\title{
A Method for Direct Estimation of Left Ventricular Global Longitudinal Strain Rate from Echocardiograms
}

\section{Brett A. Meyers}

Purdue University

Melissa C. Brindise

Purdue University

\section{Shelby Kutty}

Johns Hopkins University School of Medicine

Pavlos P. Vlachos ( $\sim$ pvlachos@purdue.edu )

Purdue University

\section{Research Article}

Keywords: Echocardiography, global longitudinal strain, image registration, speckle tracking

Posted Date: May 27th, 2021

DOI: https://doi.org/10.21203/rs.3.rs-543833/v1

License: (c) (i) This work is licensed under a Creative Commons Attribution 4.0 International License. Read Full License

Version of Record: A version of this preprint was published at Scientific Reports on March 7th, 2022. See the published version at https://doi.org/10.1038/s41598-022-06878-1. 


\section{$1 \quad$ A Method for Direct Estimation of Left 2 Ventricular Global Longitudinal Strain Rate from Echocardiograms \\ 5 Authors: \\ 6 Brett A. Meyers ${ }^{1}$, Melissa C. Brindise ${ }^{1}$, Shelby Kutty ${ }^{3}$, Pavlos P. Vlachos ${ }^{1,2 *}$ \\ 8 Affiliations: \\ 9 'School of Mechanical Engineering, Purdue University, 585 Purdue Mall, West 10 Lafayette, IN 47907, USA \\ $11{ }^{2}$ Weldon School of Biomedical Engineering, Purdue University, 206 S. Martin Jischke 12 Dr., West Lafayette, IN 47907, USA \\ $13{ }^{3}$ Taussig Heart Center, Johns Hopkins University School of Medicine, Baltimore, MD, 14 21287, USA \\ $15{ }^{*}$ Correspondence to Pavlos P. Vlachos, School of Mechanical Engineering, Purdue 16 University, pvlachos@purdue.edu \\ 17 Total word count: 4574 \\ 18}




\begin{abstract}
1 Abstract
3 We present a new method for measuring global longitudinal strain and global 4 longitudinal strain rate from 2D echocardiograms using a logarithmic-transform 5 correlation (LTC) method. Traditional echocardiography strain analysis depends on user 6 inputs and chamber segmentation, which yield increased measurement variability. In 7 contrast, our approach is automated and does not require cardiac chamber segmentation 8 and regularization, thus eliminating these issues. The algorithm was benchmarked 9 against two conventional strain analysis methods using synthetic left ventricle ultrasound 10 images. Measurement error was assessed as a function of contrast-to-noise ratio (CNR) 11 using mean absolute error and root-mean-square error. LTC showed better agreement to 12 the ground truth for strain $\left(\boldsymbol{R}^{2}=\mathbf{0 . 9 1}\right)$ and strain rate $\left(\boldsymbol{R}^{2}=\mathbf{0 . 8 5}\right)$ as compared to 13 conventional algorithms (strain $\left(\boldsymbol{R}^{2}=\mathbf{0 . 7}\right.$ ), strain rate $\left(\boldsymbol{R}^{2}=\mathbf{0 . 7}\right)$ ) and was unaffected by

14 CNR. A 200\% increase in strain measurement accuracy was observed compared to the 15 conventional algorithms. Subsequently, we tested the method using a 54-subject clinical 16 cohort (20 subjects diseased with cardiomyopathy, 34 healthy controls). Our method 17 distinguished between normal and abnormal left ventricular function with an $A \cup C=0.85$, 18 a $10 \%$ improvement over the conventional GLS algorithms.
\end{abstract}

20 Key terms

22 Echocardiography, global longitudinal strain, image registration, speckle tracking. 


\section{Introduction}

2 Global longitudinal strain (GLS), average strain of the cardiac chamber wall measured using speckle tracking echocardiography (STE), is used for the quantification of left

4 ventricle (LV) function. Because GLS is more robust to reader error than LV ejection 5 fraction ${ }^{1,2}$, it is increasingly used more in clinical practice ${ }^{3}$, especially for the detection of 6 systolic dysfunction ${ }^{4}$. Moreover, the global longitudinal strain rate (GLSr), which is 7 computed by differentiating GLS temporally, quantifies the rate of LV contraction and 8 relaxation, thus providing information on systolic and diastolic function.

9 Limitations of STE can impact GLS and GLSr measurement accuracy. In order to 10 perform a GLS-STE measurement, the user must provide an initial shape model of the 11 LV boundary, thus introducing variability and reducing measurement reproducibility ${ }^{5}$. 12 Boundary tracking is subsequently performed using block-matching or cross-correlation 13 kernels $^{6,7}$, sensitive to image quality, spatial and temporal resolution, and signal dropout ${ }^{8}$.

14 Furthermore, commercial tools use proprietary tracking and post-processing algorithms, 15 making cross-platform comparison impractical ${ }^{5,9}$. Collectively these limitations hinder wide-spread acceptance of GLS and GLSr as diagnostic parameters with established 17 standard ranges for normal and abnormal function ${ }^{1,4,10-12}$.

18 Here we present a novel algorithm for direct GLS estimation from echocardiograms 19 that overcomes the issues mentioned above. Our approach does not require LV boundary 20 initialization, regional smoothing, or assumptions of LV shape. The algorithm directly 21 measures the GLSr of the entire LV, which is then integrated in time to provide the GLS. 22 Hence, this method is user-independent and robust to noise and image artifacts. Error 23 analysis was performed using synthetic ultrasound images ${ }^{13}$ and clinical demonstration 24 was performed with patient data from healthy and cardiomyopathy subjects. In both 25 cases, we compared the results from our algorithm against conventional STE algorithms.

\section{Materials and Methods}

\section{Theory}

28 During each heartbeat, the LV undergoes complex, three-dimensional motion as it contracts and relaxes. This motion is composed of a planar translation and deformation that relate how positions $\left(x_{n}, y_{n}, z_{n}\right)$ along the LV move to $\left(x_{n+1}, y_{n+1}, z_{n+1}\right)^{14}$, 


$$
\left[\begin{array}{l}
x_{n+1} \\
y_{n+1} \\
z_{n+1}
\end{array}\right]=\boldsymbol{F}\left[\begin{array}{l}
x_{n} \\
y_{n} \\
z_{n}
\end{array}\right]+\overrightarrow{\boldsymbol{T}}=\left[\begin{array}{lll}
a_{11} & a_{12} & a_{13} \\
a_{21} & a_{22} & a_{23} \\
a_{31} & a_{32} & a_{33}
\end{array}\right]\left[\begin{array}{l}
x_{n} \\
y_{n} \\
z_{n}
\end{array}\right]+\left[\begin{array}{l}
t_{1} \\
t_{2} \\
t_{3}
\end{array}\right] .
$$

$1 \quad \overrightarrow{\boldsymbol{T}}$ is the translation matrix, and $\boldsymbol{F}$ is the deformation gradient tensor, which is related to

2 the displacement gradient tensor $\nabla \boldsymbol{u}$, as,

$$
\boldsymbol{F}=\boldsymbol{I}-\nabla \boldsymbol{u}
$$

3 where $\boldsymbol{I}$ is the identity matrix.

$4 \quad$ Lagrange strain, $\boldsymbol{\varepsilon}$, is expressed as a function of $\nabla \boldsymbol{u}$ when motion and deformation are 5 small, such that,

$$
\varepsilon=\frac{1}{2}\left(\nabla \boldsymbol{u}+\nabla \boldsymbol{u}^{T}\right)
$$

6 Equation 3 can be written as the Lagrange strain equation,

$$
\varepsilon=\frac{l-l_{0}}{l_{0}}
$$

$7 \quad$ where $l_{0}$ is the reference length and $l$ is the deformed length. The quantity $\varepsilon$ is the

8 accepted definition of $\mathrm{GLS}^{9}$. As a result, GLS is a function of the deformation gradient

9 tensor $\boldsymbol{F}$. In the following sections, we describe how we estimate GLS from the cross-

10 correlation of two consecutive images.

\section{Pairwise Cross-Correlation}

12 Image cross-correlation provides a statistical estimate of the translation of an image 13 pattern between two frames. This method is used in image registration ${ }^{15}$, speckle 14 tracking $^{16}$, particle image velocimetry ${ }^{17}$, and image correlation ${ }^{18}$. The $2 \mathrm{D}$ discrete spatial 15 cross-correlation between two images, $I_{n}$ and $I_{n+1}$, is expressed as,

$$
R(x, y)=\sum_{i=-N / 2}^{N / 2} \sum_{j=-M / 2}^{M / 2} I_{n}(i, j) I_{n+1}(x+i, y+j)
$$

16 where $R(x, y)$ is the correlation plane, $(i, j)$ are the summation indices of the correlation,

17 and $N$ and $M$ are the image height and width, respectively. The cross-correlation can be

18 performed in the spectral domain, as,

$$
R(x, y)=\mathcal{F}^{-1}\left[\overline{\mathcal{F}}\left(I_{n}(x, y)\right) \mathcal{F}\left(I_{n+1}(x, y)\right)\right],
$$

19 where $\mathcal{F}$ is the $2 \mathrm{D}$ Fourier transformation $(\mathrm{FT})$ and $\overline{\mathcal{F}}$ is the complex conjugate of the 20 FT. The expanded form of Equation 6 is written as, 


$$
R(x, y)=\iint \bar{G}_{n}(u, v) G_{n+1}(u, v) e^{-j 2 \pi\left(u\left(t_{1}+x\right)+v\left(t_{2}+y\right)\right)} d u d v,
$$

where $(u, v)$ are wavenumbers proportional to positions $(x, y)$ and $G$ is the image FT.

The Fourier transform affine theorem stipulates that rotation, stretch, and shear occurs

3 on the FT magnitude and phase ${ }^{19}$. We use this to establish how the affine transform

4 affects the rigid translation estimate by replacing $G_{n+1}$ in Equation 7 with the relationship

5 for $G_{n}$ using Equation 1,

$$
R(x, y)=\iint \frac{\bar{G}_{n}(u, v) G_{n}\left(u^{\prime}, v^{\prime}\right)}{|\boldsymbol{F}|} e^{-j 2 \pi\left(u^{\prime}\left(t_{1}+x\right)+v^{\prime}\left(t_{2+y}\right)\right)} d u d v .
$$

6 Here, $u^{\prime}=a_{11} u+a_{21} v, v^{\prime}=a_{12} u+a_{22} v$, and $|\boldsymbol{F}|=\operatorname{det}(\boldsymbol{F})$. Equation 8 provides a

7 correlation plane with the peak shifted from the plane center by $(\Delta x, \Delta y)$, directly related

8 to the translations $t_{1}$ and $t_{2}$ and the local deformation gradient tensor $\boldsymbol{F}$. The correlation 9 peak shifts are written as,

$$
\Delta x \cong a_{11} t_{1}+a_{12} t_{2}, \quad \Delta y \cong a_{21} t_{1}+a_{22} t_{2} .
$$

10 The deformation gradients $a_{i j}$ produce the strain captured by the GLS measurement.

\section{Translation-Invariant FT Magnitude Correlation for GLS Estimation}

12 The components of $\boldsymbol{F}$ can be estimated separately of $\overrightarrow{\boldsymbol{T}}$ using the magnitude, $|F(u, v)|$,

13 of the $\mathrm{FT}^{19,20}$. The cross-correlation of the $\mathrm{FT}$ magnitudes is translation invariant yielding

14 the terms of $\boldsymbol{F}$ with no contribution from $\overrightarrow{\boldsymbol{T}}$. The log-polar basis Fourier-Mellin 15 transform ${ }^{21,22}$, popular in image registration, decouples terms of $\boldsymbol{F}$ to estimate image 16 rotation and stretch.

17 Contraction and relaxation of the LV result in deformation akin to anisotropic image 18 rescaling. By changing the FT magnitude image coordinates from cartesian $(u, v)$ to 19 orthogonal logarithmic coordinates $(\log u, \log v)$, the resulting displacements from the 20 correlation between two FT magnitude images now correspond to horizontal $\Delta u$ and 21 vertical $\Delta u$ rescaling such that,

$$
G(\log u+\log \Delta u, \log v+\log \Delta v) .
$$

22 This correlation is affected by rotation and shear based on the terms present in 23 Equation 8. However, if those terms are minimized beforehand, the correlation peak shift 24 estimates $(\Delta u, \Delta v)$ can be related to the terms $a_{11}$ and $a_{22}$ from $\boldsymbol{F}$ through, 


$$
a_{11}=\Delta u \cong e^{\Delta x}, \quad a_{22}=\Delta v \cong e^{\Delta y}
$$

1 We substitute $a_{11}$ and $a_{22}$ from Equation 11 into $\boldsymbol{F}$ in Equation 2 and solve for $\nabla \mathbf{u}$ such 2 that,

$$
\nabla \mathbf{u}=\left[\begin{array}{cc}
e^{d x}-1 & 0 \\
0 & e^{d y}-1
\end{array}\right]
$$

3 Since deformation of the LV in long axis apical (ALAX) scans occurs along its length, 4 we assume GLS occurs predominantly along the vertical direction, providing the GLS 5 estimator,

$$
\varepsilon_{G L S}=\frac{l-l_{0}}{l_{0}} \approx e^{d y}-1 \text {. }
$$

\section{Direct Global Longitudinal Strain Estimation Algorithm}

We now describe our algorithm using the translation-invariant FT magnitude correlation to estimate the GLS based on Equation 13. A schematic of our algorithm is

9 provided in Figure 1. The algorithm comprises two stages - the first performs an image registration to minimize shear and rotation that corrupt the correlation accuracy, while the

11 second performs the GLS estimation.

12 The algorithm begins by selecting frames to analyze (Figure 1a). Next, the user selects 13 three points from the first frame (Figure 1b), corresponding to approximate locations of

14 the LV apex, the annulus septal, and annulus lateral positions. These points are tracked 15 between consecutive frames using standard pairwise cross-correlation (Figure 1c). For 16 each frame, the geometric center from the tracked points is computed along with the 17 vertical axis's orientation angle for a line formed from the annulus center to the apex 18 (Figure 1d). Frames are then aligned based on the geometric center, and the orientation 19 angle is corrected. A circular ROI for each frame is defined from the tracked points.

20 In the second stage, for each pair of sequential registered images, $t$ and $t+1$ (Figure 21 1e), their FT and FT magnitude is computed. The FT magnitudes are interpolated from 22 the image grid onto a logarithm-scale grid (Figure 1f). Because the FT logarithm 23 transformed images are symmetric about the image diagonals, they are separated into 24 four quarters to improve measurement accuracy. Each sub-image is filtered ${ }^{23}$ and their 25 FT is computed (Figure 1g). The FT sub-images are then correlated using the spectral 26 cross-correlation kernel and ensemble-averaged to provide the displacements $(\Delta x, \Delta y)$ 
1 (Figure 1h). A dynamic phase-filtered kernel is applied to the cross-correlation to improve 2 estimate accuracy ${ }^{24-26}$. The displacements $(\Delta x, \Delta y)$ are adjusted based on the logarithm-

3 scale grid, becoming $\left(\Delta \mathrm{x}^{\prime}, \Delta \mathrm{y}^{\prime}\right)$. The GLSr between frames is computed using $\Delta \mathrm{y}^{\prime}$ and

4 Equation 13. Finally, GLSr across each frame pair is integrated in time using $4^{\text {th }}$-Order

5 Runge-Kutta to obtain GLS (Figure 1i). The integral operator provides inherent 6 smoothing, which suppresses noise in the GLS measurements. We will hereafter refer to 7 this method as the Logarithm-Transform Correlation (LTC) method.

\section{Speckle Tracking Strain}

9 This study uses two standard STE algorithms against which we benchmark our 10 method. One algorithm uses the spatial cross-correlation kernel introduced in Equation $115^{16}$, referred to herein as the Direct Cross-Correlation or DCC method. The second uses 12 the spectral cross-correlation (Equation 6), hereafter referred to as the Fourier Transform 13 Correlation or FTC method.

14 Boundary tracking was performed by propagating the segmented boundary of the initial 15 frame through the estimated displacement fields using $4^{\text {th }}$-Order Runge-Kutta. GLS was 16 estimated as the measured change in arc-length between the segmented and the tracked 17 boundary from each frame. Image co-registration was not performed, as it is not required 18 to obtain a consistent GLS measurement based on the arc-length change calculation.

\section{Artificial Echocardiograms}

20 Error analysis was performed using synthetic LV ALAX echocardiograms ${ }^{13,27}$. Ground 21 truth boundaries, displacements, and strains for each dataset were included with the 22 synthetic images. Mean absolute error (MAE) and root-mean-square error (RMSE) for 23 GLS and GLSr were quantified as a function of contrast to noise ratio (CNR). CNR was 24 defined as the ratio of L2-norm of the tissue signal to L2-norm of the signal inside the $25 \mathrm{LV}^{28}$. Error quantities were normalized by the peak GLS or peak GLSr.

\section{Clinical Imaging}

27 The method's clinical capabilities were demonstrated using a cohort of pediatric 28 patients with confirmed cardiomyopathy and age-matched controls collected from a study 29 conducted at the University of Nebraska Medical Center in Omaha, Nebraska, USA. The 30 Institutional Review Boards of Purdue, Nebraska, and Johns Hopkins Universities each 31 approved the study protocol. All procedures were performed in accordance with relevant 
1 guidelines and regulations. Informed written consent was obtained from study subject 2 guardians for those under age 18 or from the subject themself for those over age 18.

3 Each patient underwent a routine echocardiogram study on an iE33 ultrasound system

4 (Philips Healthcare, Andover, MA, USA). Studies were collected based on the American 5 Society of Echocardiography recommendations ${ }^{29}$. The 54 -subject cohort included 4 6 patients with confirmed dilated cardiomyopathy (DCM), 16 patients with confirmed 7 hypertrophic cardiomyopathy ( $\mathrm{HCM})$, and 34 age-matched controls. Information on the 8 cohort demographics is provided in Table 1 and heart function indices in Table 2.

9 Doppler measurements were collected in the ALAX A4C view. B-mode ALAX A2C, $\mathrm{A} 3 \mathrm{C}$, and $\mathrm{A} 4 \mathrm{C}$ view scans were performed conventionally, not explicitly collected for

11 strain measurements. Images were stored in Digital Imaging and Communications in

12 Medicine (DICOM) format for post-processing. 900 individual heartbeats were analyzed, 13 consisting of 573 control, $261 \mathrm{HCM}$, and $66 \mathrm{DCM}$. Peak absolute GLS, peak absolute 14 systolic GLSr (GLSrs), and peak absolute early diastolic GLSr (GLSre) were computed. 15 Ventricle dimension measurements were computed by the Simpson biplane method using 16 the GE EchoPAC software. Data were classified into control (CTRL) and cardiomyopathy 17 (CM) groups. Statistical significance was tested using Student's t-test between methods 18 among the same condition and between each condition. Receiver operating characteristic 19 (ROC) curves and area under the curve (AUC) were computed for each parameter.

\section{Results}

\section{Error Analysis Results}

22 Figure 2 presents error analysis results. The GLSr estimates (Figure 2a-1) by the DCC 23 and FTC method yielded linear regression fits with similar slopes and bias of 24 approximately $m \approx 0.7 \mathrm{~s} \cdot \mathrm{s}^{-1}$ and $b \approx 0.025 \mathrm{~s}^{-1}$, and quality of fit of $R_{D C C}^{2}=0.72$ and $25 R_{F T C}^{2}=0.76$. Conversely, LTC GLSr estimates had a slope fit of $m=0.92 \mathrm{~s} \cdot \mathrm{s}^{-1}$ and bias 26 of $b=0.016 \mathrm{~s}^{-1}$ with a fit quality of $R_{L T C}^{2}=0.85$. GLSr, as a function of CNR, is shown in

27 Figure 2a-2 (values are normalized by $G L S r=0.95 s^{-1}$ ). LTC showed a 1.5 to 2-fold 28 improvement in accuracy than DCC and a 1.5-fold improvement compared to FTC. 29 Additionally, the LTC method is unaffected by CNR. In contrast, the FTC and DCC 30 methods show a CNR dependence, albeit less for the FTC method. 
GLS estimates by the DCC and FTC method maintained linear regression fits (Figure $2 \mathrm{~b}-1$ ) with similar slopes and biases of around $m \approx 0.6$ and $b \approx 0.3 \%$, and quality of fit of $R_{D C C}^{2}=0.71$ and $R_{F T C}^{2}=0.74$. LTC GLS estimates show a slope of $m=0.92$, a bias of $b=$

$4 \quad 0.09 \%$, and fit quality of $R_{L T C}^{2}=0.91$. The LTC method shows more than $200 \%$ improvement

5 in measurement accuracy compared against the DCC and FTC methods as a function of

6 CNR (Figure 2b-2), where values are normalized by $G L S=8.47 \%$. The error analysis

7 demonstrates that the LTC method is unaffected by CNR, while the DCC and FTC 8 methods are affected by signal quality.

\section{Clinical Analysis Results}

10 Results comparing the LTC method against conventional GLS methods for each 11 parameter are presented in Figure 3a-1 through 3c-1. LTC consistently measured 12 increased peak GLS, GLSrs, and GLSre, compared to the conventional methods. FTC 13 mean peak GLS measurements were increased compared to the DCC mean peak GLS. 14 However, the FTC maintained an increased variance across both health states. This 15 occurred for FTC GLSre and GLSrs, and the results are similar for the DCC method. 16 Significance tests indicated the group means were statistically significant $(p<0.05)$ for 17 all cases.

18 ROC curves are presented in Figure 3a-2 through 3c-2. The peak GLS ROC curves (Figure 3a-2) show that the LTC method AUC was 0.85 , while the FTC and DCC AUCs were near 0.75. Peak GLSrs ROC curves (Figure 3b-2) show that all methods performed similarly in detecting abnormal function for this dataset. The LTC had $p<0.05$ when compared to FTC but showed weak significance to DCC. Finally, for the peak GLSre ROC curves (Figure 3c-2), the LTC maintained an AUC of 0.82, and the FTC method had an

24 AUC of 0.75 , but both methods did not achieve the AUC from the peak GLS. Additionally,

25 LTC achieved $p<0.05$ against both methods.

\section{Discussion}

27 This study presents a new algorithm, the LTC method, for computing GLS and GLSr 28 estimates from ultrasound scans. Error analysis using synthetic ultrasound images 29 demonstrated and quantified the LTC method's improvement over two current STE 30 methods, and a clinical cohort was analyzed using the LTC and the two STE methods. 
1 The LTC method does not rely on LV shape assumptions and avoids the use of boundary 2 segmentation. Furthermore, because LV segmentation is not required, regularization to 3 preserve the segmentation shape is avoided. Lastly, because the entire image of the LV

4 is used to compute strain, out-of-plane motion is averaged out, making this method 5 uniquely robust this error source.

6 The LTC method is novel by directly computing the GLSr between sequential frames, 7 ensuring a reliable rate measurement. Computing GLS by integrating the GLSr, provides 8 a smoothing operation that reduces noise. Commercial GLS algorithms have constraints 9 that enforce tracked boundaries that are smooth in space and time, providing 10 measurements that appear physically consistent. However, the returned measurements 11 are also driven by the regularization. Thus, the GLS measurements do not adhere to the underlying deformation that occurs between frames.

13 The error analysis presented in Figure 2 demonstrated that the DCC and FTC methods 14 are dependent on CNR. As CNR approaches 1, the mean pixel intensity of the LV wall 15 and the mean pixel intensity of speckle noise are almost equal. This means the speckle 16 noise cannot be differentiated from the LV wall, making the physical features ambiguous.

17 Both methods underestimated the GLS, supporting the notion that it may be best to avoid 18 such computation ${ }^{5}$. In contrast, the LTC method enables robust GLS computation even with noisy images, as supported by the MAE and RMSE plots in Figure 2.

Comparison of each method for all disease conditions, presented in Figure 3a-1, b-1, and $\mathrm{c}-1$, provides the measurement distributions and their statistical significance. For each method, the CTRL and CM distributions overlapped while statistical significance was observed. The LTC method reported larger GLS and GLSr values compared to both STE methods. Between methods, statistical significance was observed while distributions overlapped. Comparison results suggest normal function will report larger GLS and GLSr values than abnormal function but establishing improvement of the LTC method through 27 this analysis alone is not possible.

28 The ROC curves, presented in Figure $3 a-2,3 b-2$, and $3 c-2$, are used to determine if 29 clinical separation is possible. The LTC method peak GLS and GLSre ROC curves show 30 a 10\% improvement in classification, which would improve the correct diagnosis rate. 
LTC peak GLSre measurements agreed with the literature (peak GLSre $\left.>1 \mathrm{~s}^{-1}\right)^{30}$, while GLS and GLSrs measurements were below nominal ranges from literature (peak GLS> $18 \%$; peak GLSrs $\left.>1 \mathrm{~s}^{-1}\right)^{31}$. Commercial methods rely on regularization steps, which force

4 the measurements to fit in LV shape models ${ }^{5,9}$, thereby causing the GLS measurements

5 to be a regularization function instead of the actual underlying deformation, feasibly 6 leading to overestimation.

7 LTC algorithm limitations stem from the assumption that GLS and GLSr can be reliably 8 measured from the septal and lateral walls, ignoring shortening near the apex. 9 Furthermore, the algorithm assumes that reorientation has been performed correctly with no misalignment. If misalignment exists, the measurements will be a combination of GLS

11 and off-axis strain. Finally, this study was limited by its clinical validation, which was 12 performed using retrospective pediatric data. A dataset optimized for GLS estimation 13 would help further substantiate findings. Additional testing should be performed using 14 adult heart failure subjects.

15 We presented a new correlation kernel, the logarithm transform correlation or LTC, for quantifying GLS and GLSr from echocardiography scans. Our LTC-based algorithm does

17 not use LV shape assumptions, is machine-agnostic, automated, and free of heuristic 18 inputs. We compared the LTC against STE algorithms using artificial scans, analyzing error against ground truth GLS and GLSr values, and validated using clinical data from a study of pediatric cardiomyopathies. Results showed that the LTC method is unaffected 21 by the image quality, providing improved measurement accuracy against the STE 22 methods for both the synthetic data and clinical cohort.

\section{References}

24 1. Kalam, K., Otahal, P. \& Marwick, T. H. Prognostic implications of global LV dysfunction: A systematic review and meta-analysis of global longitudinal strain and ejection fraction. Heart 100, 1673-1680 (2014).

2. Stanton, T., Leano, R. \& Marwick, T. H. Prediction of all-cause mortality from global longitudinal speckle strain: comparison with ejection fraction and wall motion scoring. Circ. Cardiovasc. Imaging 2, 356-364 (2009).

3. Lang, R. M. et al. Recommendations for chamber quantification. Eur. J. Echocardiogr. 7, 79-108 (2006). 
1 4. Smiseth, O. A., Torp, H., Opdahl, A., Haugaa, K. H. \& Urheim, S. Myocardial strain imaging: how useful is it in clinical decision making? Eur. Heart J. 37, 1196-1207 (2016).

4 5. Lang, R. M. et al. Recommendations for Cardiac Chamber Quantification by

7. Helle-Valle, T. et al. New noninvasive method for assessment of left ventricular Echocardiography in Adults: An Update from the American Society of Echocardiography and the European Association of Cardiovascular Imaging. J. Am. Soc. Echocardiogr. 28, 1-39.e14 (2015).

6. Amundsen, B. H. et al. Noninvasive Myocardial Strain Measurement by Speckle Tracking Echocardiography. J. Am. Coll. Cardiol. 47, 789-793 (2006). rotation: speckle tracking echocardiography. Circulation 112, 3149-3156 (2005).

8. Rösner, A. et al. The influence of frame rate on two-dimensional speckle-tracking strain measurements: a study on silico-simulated models and images recorded in patients. Eur. Hear. Journal-Cardiovascular Imaging 16, 1137-1147 (2015).

9. Voigt, J. U. et al. Definitions for a common standard for 2D speckle tracking echocardiography: consensus document of the EACVI/ASE/Industry Task Force to standardize deformation imaging. Eur. Hear. J. - Cardiovasc. Imaging 16, 1-11 (2015).

10. Farsalinos, K. E. et al. Head-to-Head Comparison of Global Longitudinal Strain Measurements among Nine Different Vendors: The EACVI/ASE Inter-Vendor Comparison Study. J. Am. Soc. Echocardiogr. 28, 1171-1181.e2 (2015).

11. Yingchoncharoen, T., Agarwal, S., Popović, Z. B. \& Marwick, T. H. Normal Ranges of Left Ventricular Strain: A Meta-Analysis. J. Am. Soc. Echocardiogr. 26, 185-191 (2013).

12. Menting, M. E. et al. Normal myocardial strain values using $2 \mathrm{D}$ speckle tracking echocardiography in healthy adults aged 20 to 72 years. Echocardiography 33, 1665-1675 (2016).

13. Alessandrini, M. et al. Realistic Vendor-Specific Synthetic Ultrasound Data for Quality Assurance of 2-D Speckle Tracking Echocardiography: Simulation Pipeline and Open Access Database. IEEE Trans. Ultrason. Ferroelectr. Freq. Control 65, 411-422 (2018). 
1 14. Meunier, J. \& Bertrand, M. Echographic Image Mean Gray Level Changes with Tissue Dynamics: A System-Based Model Study. IEEE Trans. Biomed. Eng. 42, 403-410 (1995).

4 15. Anuta, P. E. Spatial registration of multispectral and multitemporal digital imagery using fast fourier transform techniques. IEEE Trans. Geosci. Electron. 8, 353-368 (1970).

16. Bohs, L. N. \& Trahey, G. E. A Novel Method for Angle Independent Ultrasonic Imaging of Blood Flow and Tissue Motion. IEEE Trans. Biomed. Eng. 38, 280-286 (1991).

17. Willert, C. E. \& Gharib, M. Digital Particle Image Velocimetry. Exp. Fluids 10, 181193 (1991).

18. Chu, T. C., Ranson, W. F. \& Sutton, M. a. Applications of Digital Image Correlation Techniques to Experimental Mechanics. Exp. Mech. 25, 232-244 (1985).

19. Bracewell, R. N., Chang, K.-Y., Jha, A. K. \& Wang, Y.-H. Affine theorem for twodimensional Fourier transform. Electron. Lett. 29, 304-304 (1993).

21. Chen, Q., Defrise, M. \& Deconinck, F. Symmetric Phase-Only Matched Filtering of Fourier-Mellin Transforms for Image Registration and Recognition. IEEE Trans. Pattern Anal. Mach. Intell. 16, 1156-1168 (1994).

20. Reddy, B. S. \& Chatterji, B. N. An FFT-based technique for translation, rotation, and scale-invariant image registration. IEEE Trans. image Process. 5, 1266-1271 (1996).

22. Giarra, M. N., Charonko, J. J. \& Vlachos, P. P. Measurement of fluid rotation, dilation, and displacement in particle image velocimetry using a Fourier-Mellin cross-correlation. Meas. Sci. Technol. 26, 35301 (2015).

23. Eckstein, A. \& Vlachos, P. P. Assessment of advanced windowing techniques for digital particle image velocimetry (DPIV). Meas. Sci. Technol. 20, 075402 (2009).

24. Meyers, B. A., Goergen, C. J. \& Vlachos, P. P. Development and Validation of a Phase-Filtered Moving Ensemble Correlation for Echocardiographic Particle Image Velocimetry. Ultrasound Med. Biol. 44, 477-488 (2018).

25. Eckstein, A. \& Vlachos, P. P. Digital particle image velocimetry (DPIV) robust phase correlation. Meas. Sci. Technol. 20, 055401 (2009). 
1 26. Eckstein, A. C., Charonko, J. \& Vlachos, P. Phase correlation processing for DPIV measurements. Exp. Fluids 45, 485-500 (2008).

27. Alessandrini, M. et al. Generation of ultra-realistic synthetic echocardiographic sequences to facilitate standardization of deformation imaging. in 2015 IEEE 12th International Symposium on Biomedical Imaging (ISBI) vols 2015-July 756-759 (IEEE, 2015).

28. Bell, M. A. L., Goswami, R., Kisslo, J. A., Dahl, J. J. \& Trahey, G. E. Short-lag spatial coherence imaging of cardiac ultrasound data: Initial clinical results. Ultrasound Med. Biol. 39, 1861-1874 (2013).

29. Lopez, L. et al. Recommendations for quantification methods during the performance of a pediatric echocardiogram: a report from the Pediatric Measurements Writing Group of the American Society of Echocardiography Pediatric and Congenital Heart Disease Council. J. Am. Soc. Echocardiogr. 23, 465-495 (2010).

31. Jashari, H. et al. Normal ranges of left ventricular strain in children: a meta-analysis. Cardiovasc. Ultrasound 13, 1021 (2015).

\section{Acknowledgments}

The authors thank Mary J. Craft for her time and effort in data collection, aggregation, and sharing between universities. The support of CTSI and NIH (UL1TR002529) is gratefully acknowledged.

\section{Author Contributions}

25 BAM, MCB, and PPV designed the algorithms. SK designed the study and coordinated 26 subject enrollment. BAM performed the data analysis. BAM wrote the paper and all other 27 authors made significant contributions to the writing.

\section{Funding}

29 This project was supported by the Indiana Clinical and Translational Sciences Institute 30 and funded by Grant Number UL1TR002529 from the National Institutes of Health, 
1 National Center for Advancing Translational Sciences, Clinical, and Translational

2 Sciences Award.

3 Competing interests

4 BAM and PPV are employees of Cordian Technologies, a company having a portfolio 5 of patents for B-mode and Doppler echocardiogram-based measurements. MCB and SK 6 have no potential conflict of interest. 
Figure Legends

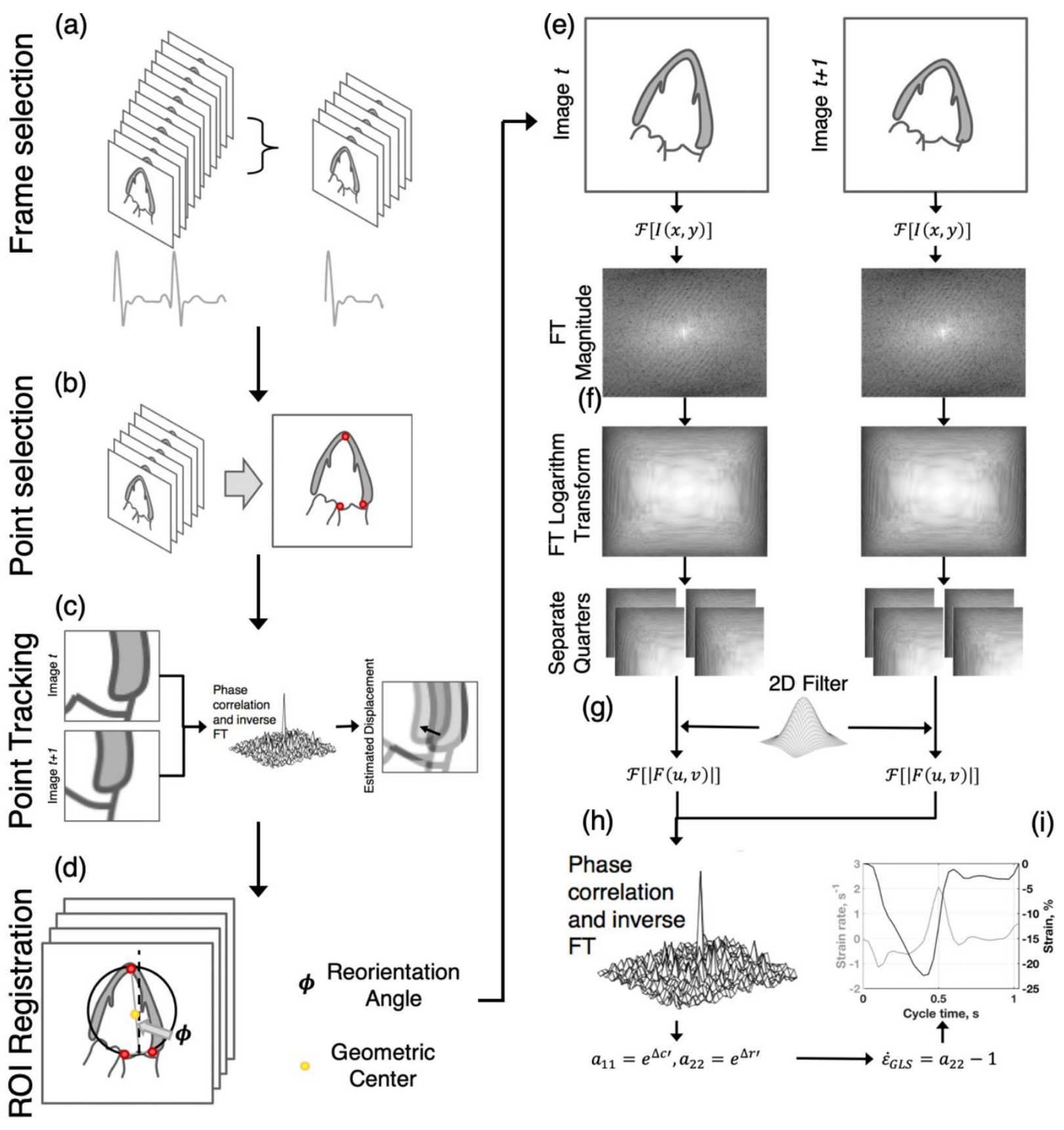

$4 \quad$ Figure 1: Illustration of the direct global longitudinal strain estimation algorithm. (a) 5 Frames for a single beat are selected from an input echocardiogram. (b) User inputs for 6 the apex and annulus positions from a reference frame are provided. (c) The inputs are 7 tracked temporally. (d) Frame co-registration is performed. (e) The LV is cropped from 8 each frame, and these sub-images are Fourier-transformed. (f) The FT magnitude is 
1 calculated, interpolated onto a logarithm-basis, and separated into four sub-images. (g)

2 Each sub-image is Fourier-transformed and convolved with a phase filter. (h) Ensemble

3 phase correlation is performed, producing a correlation plane with a peak shifted from the

4 plane center. This shift corresponds to a frame pair strain rate. (i) Strain is computed by

5 temporally integrating the strain rate estimates.

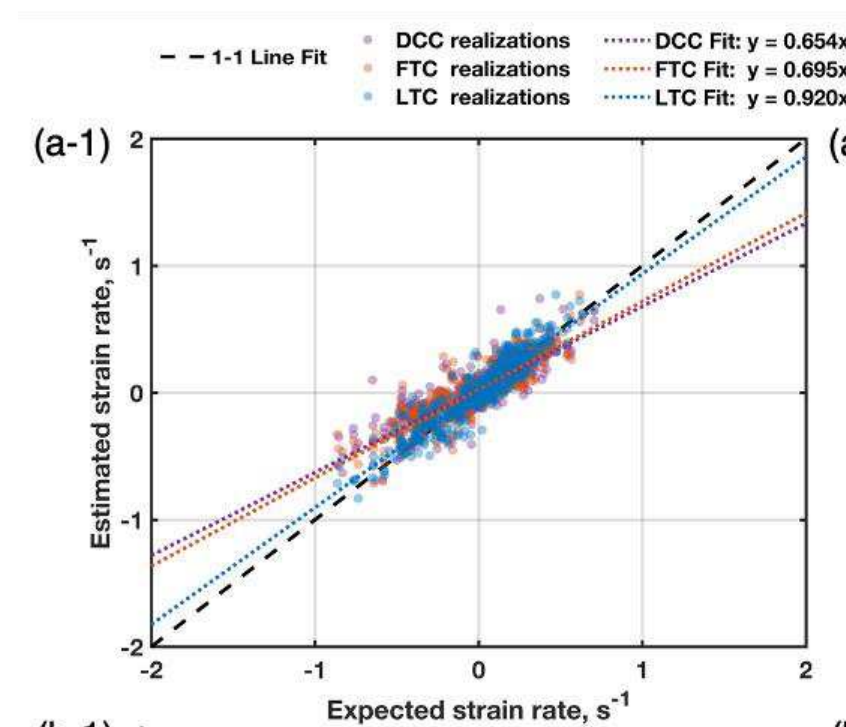

(b-1)

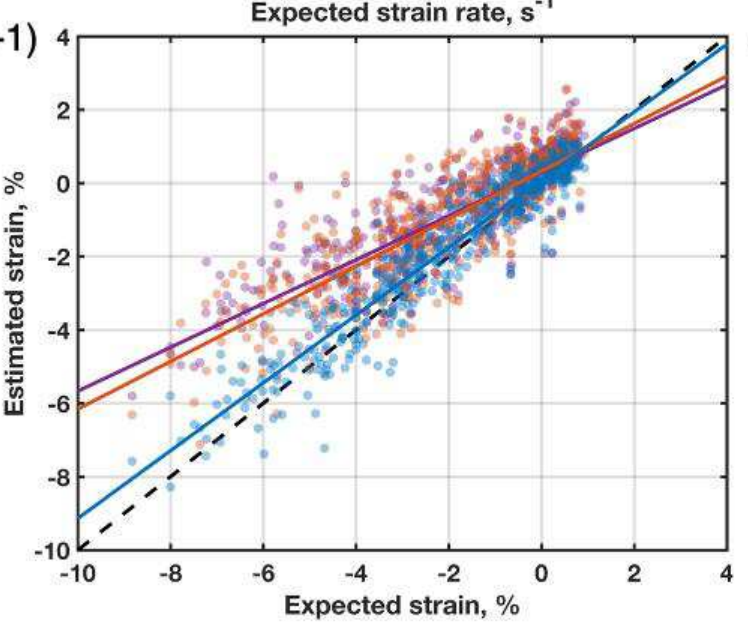

(b-2)

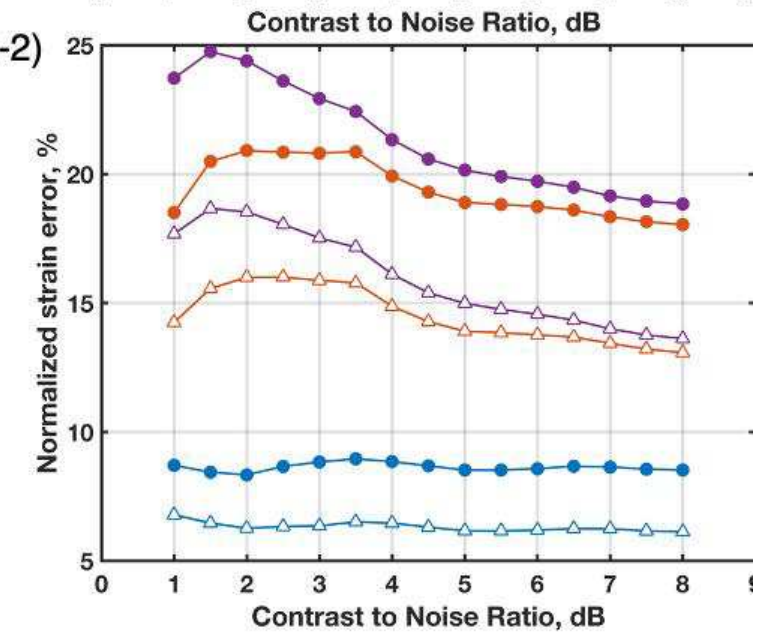

Figure 2: (Left) Direct comparison of measurements to ground truth values and (right)

8 normalized mean absolute error (MAE) and root mean square error (RMSE) as a function

9 of contrast-to-noise ratio (CNR) for (a) GLSr and (b) GLS quantities. Measurements were

10 performed using the Direct Cross-Correlation method (DCC), Fourier Transform

11 Correlation (FTC), and Fourier-based Logarithm Transform Correlation (LTC). 

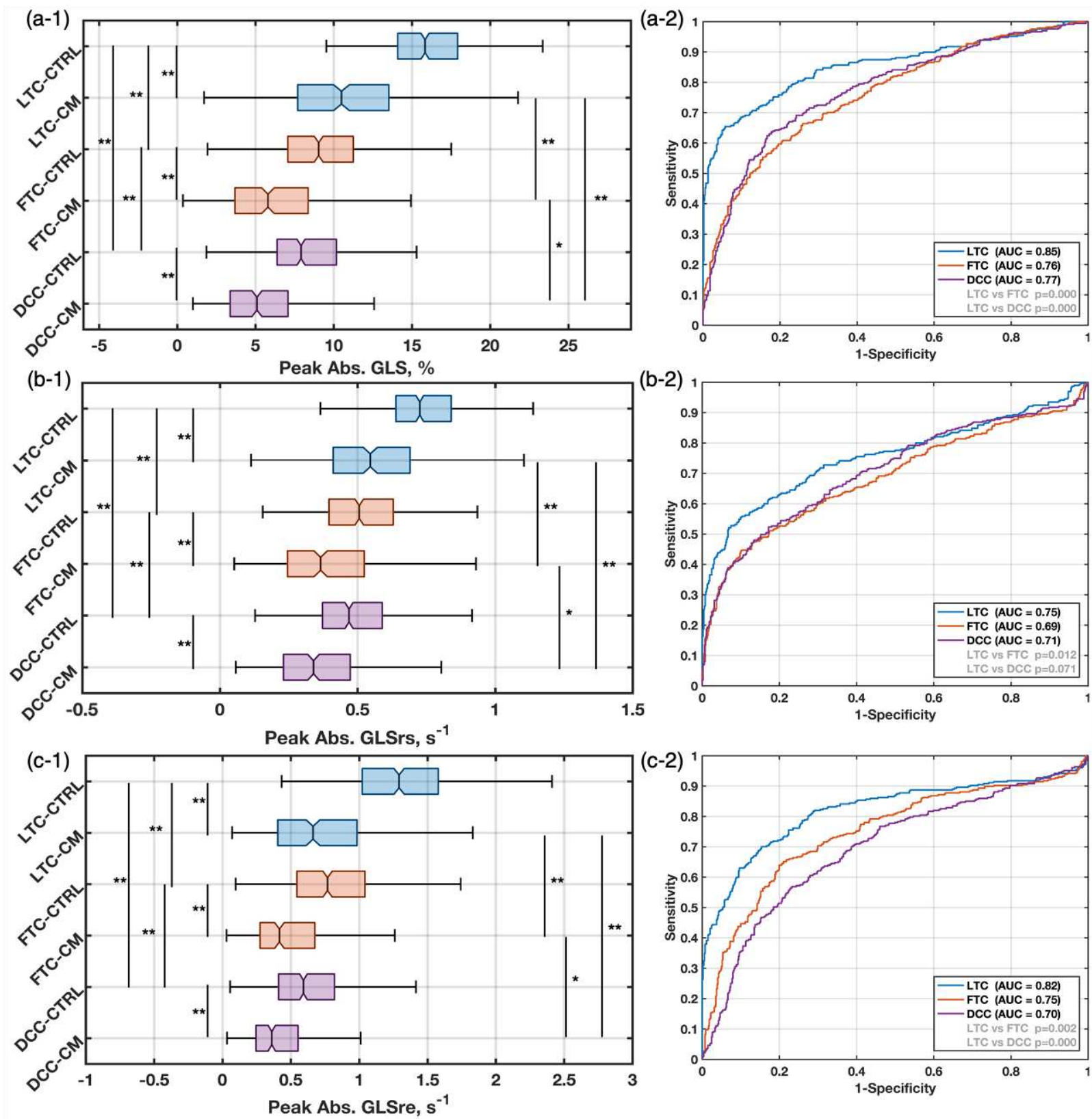

NS - Not Significant, * $-p<0.050,{ }^{\star \star}-p<0.001$

2 Figure 3: Distribution of measurements and significance tests for each GLS

3 measurement method on observing (a-1) peak absolute GLS, (b-1) peak absolute systolic

$4 \mathrm{GLSr}$, and (c-1) peak absolute diastolic GLSr. (2) Receiver operating characteristic (ROC)

5 curves displaying the ability for the LTC method estimated parameters to distinguish

6 between normal and abnormal cardiac disease states based on (a-2) peak absolute GLS,

7 (b-2) peak absolute systolic GLSr, and (c-2) peak absolute diastolic GLSr. The analysis

8 was performed on a set of control subjects (CTRL) and subjects with cardiomyopathies

9 (CM). 


\section{$1 \quad$ Tables and Table Legends}

2 Table 1: Demographics of the study cohort for each disease state.

\begin{tabular}{lrrr}
\hline Characteristics & $\begin{array}{r}\text { Control } \\
(n=33)\end{array}$ & $\begin{array}{r}\text { DCM } \\
(n=4)\end{array}$ & $\begin{array}{r}\text { HCM } \\
(n=16)\end{array}$ \\
\hline Age (years) & $17.98 \pm 8.86$ & $14.50 \pm 6.24$ & $18.74 \pm 10.47$ \\
BSA $\left(\mathrm{m}^{2}\right)$ & $1.66 \pm 0.56$ & $1.52 \pm 0.64$ & $1.81 \pm 0.69$ \\
Height $(\mathrm{cm})$ & $159.25 \pm 29.11$ & $147.90 \pm 57.60$ & $159.81 \pm 30.86$ \\
Weight $(\mathrm{kg})$ & $63.50 \pm 30.42$ & $57.60 \pm 35.65$ & $75.71 \pm 41.03$ \\
Heart Rate $(\mathrm{bpm})$ & $67.47 \pm 17.26$ & $92.50 \pm 33.81$ & $72.88 \pm 18.78$ \\
\hline
\end{tabular}

$4 \quad$ Table 2: Indices for LV dimensions and functional parameters.

\begin{tabular}{|c|c|c|c|}
\hline Ventricular Dimensions & $\begin{array}{c}\text { Control } \\
(n=33)\end{array}$ & $\begin{array}{r}\mathrm{DCM} \\
(n=4)\end{array}$ & $\begin{array}{r}\mathrm{HCM} \\
(n=16)\end{array}$ \\
\hline End Diastolic Volume (ml) & $98.85 \pm 39.19$ & $178.75 \pm 83.92$ & $96.28 \pm 37.66$ \\
\hline End Systolic Volume (ml) & $37.80 \pm 15.84$ & $117.75 \pm 60.31$ & $36.03 \pm 18.40$ \\
\hline Stroke Volume (ml) & $61.29 \pm 24.39$ & $61.00 \pm 33.32$ & $59.44 \pm 21.24$ \\
\hline Ejection Fraction (\%) & $62.16 \pm 3.50$ & $34.25 \pm 14.93$ & $63.06 \pm 6.01$ \\
\hline \multicolumn{4}{|l|}{ Functional Parameters } \\
\hline E-wave velocity $\left(\mathrm{cm} \mathrm{s}^{-1}\right)$ & $82.20 \pm 19.70$ & $102.25 \pm 29.80$ & $83.19 \pm 19.59$ \\
\hline A-wave velocity $\left(\mathrm{cm} \mathrm{s}^{-1}\right)$ & $42.84 \pm 10.95$ & $61.25 \pm 34.74$ & $61.13 \pm 33.65$ \\
\hline $\mathrm{e}^{\prime}$ velocity $\left(\mathrm{cm} \mathrm{s}^{-1}\right)$ & $17.58 \pm 3.22$ & $11.38 \pm 2.63$ & $10.51 \pm 3.04$ \\
\hline E/A ratio & $2.00 \pm 0.59$ & $2.24 \pm 1.62$ & $1.55 \pm 0.50$ \\
\hline E/e' ratio & $4.06 \pm 1.21$ & $8.22 \pm 4.30$ & $7.34 \pm 2.58$ \\
\hline
\end{tabular}


(a)

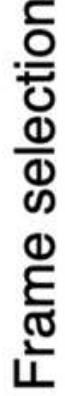

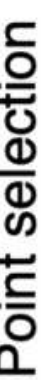

(c)

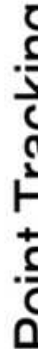

돈
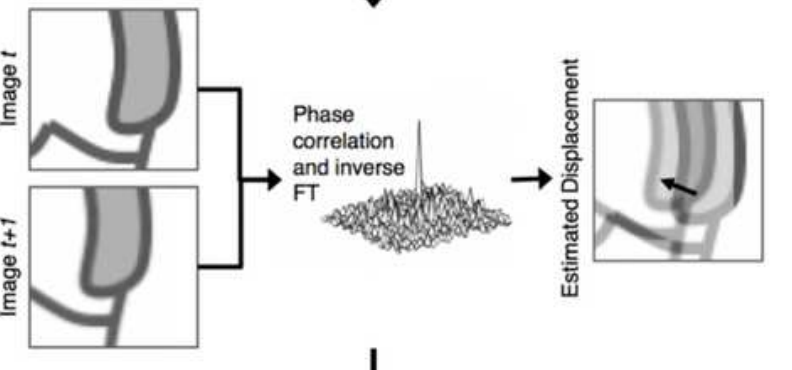

(d)

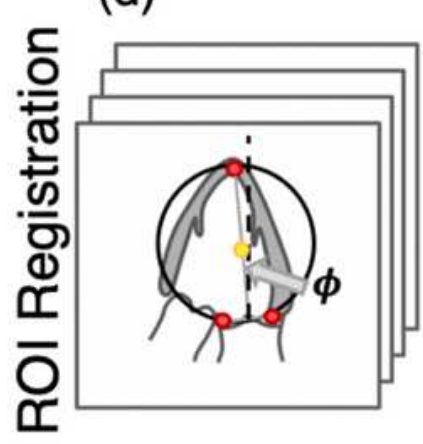

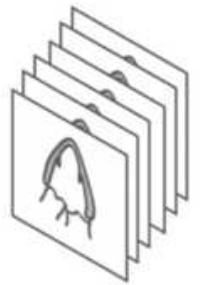
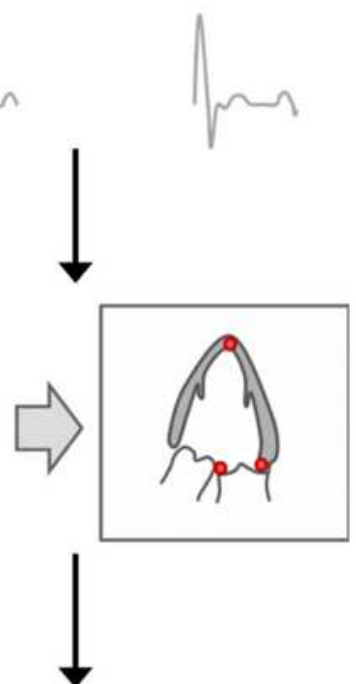

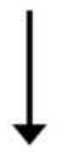

(e)
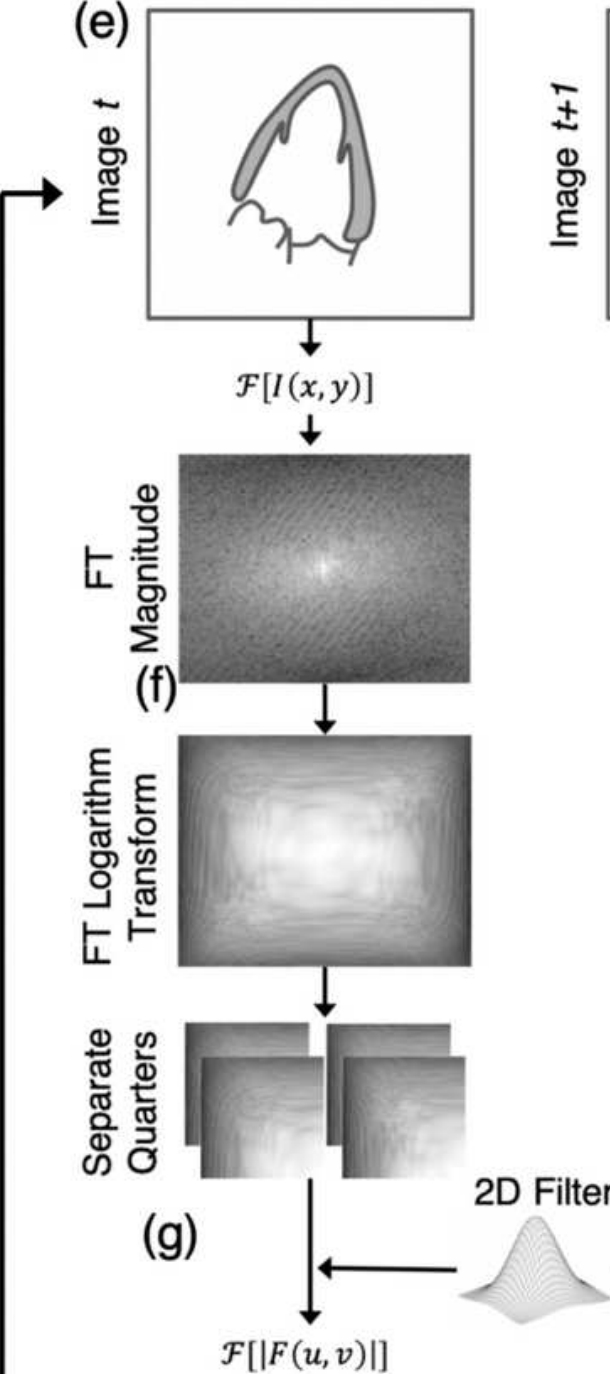

(h)
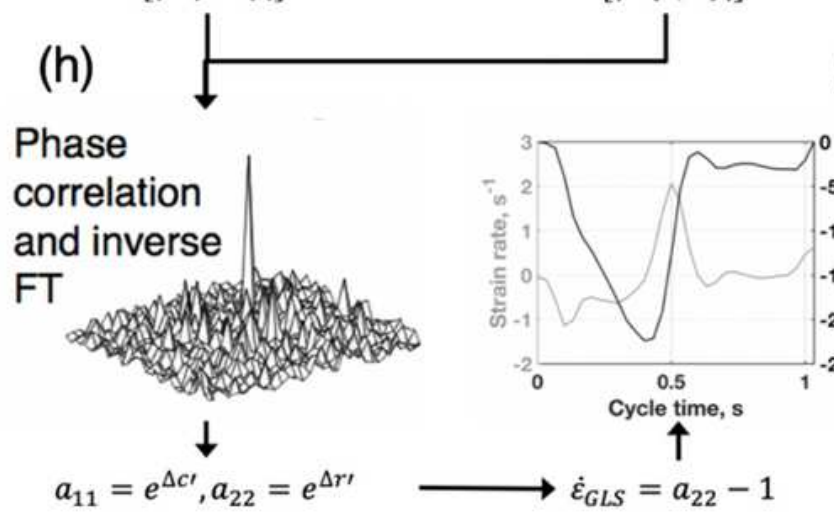

(i)

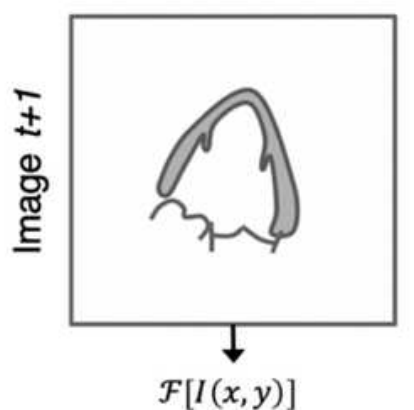

$\downarrow$
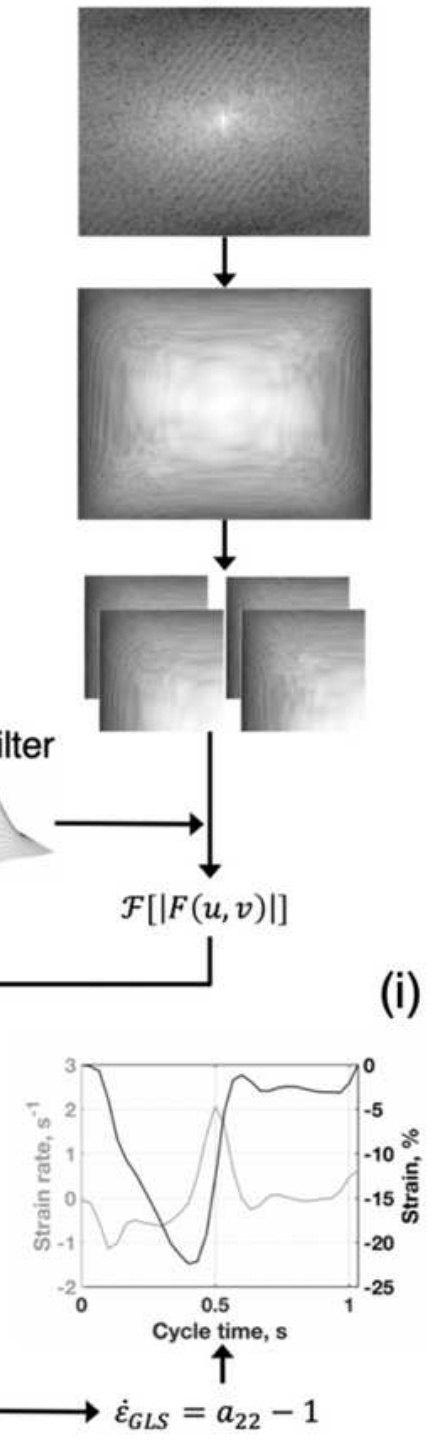

Figure 1

Illustration of the direct global longitudinal strain estimation algorithm. (a) Frames for a single beat are selected from an input echocardiogram. (b) User inputs for the apex and annulus positions from a reference frame are provided. (c) The inputs are tracked temporally. (d) Frame co-registration is 
performed. (e) The LV is cropped from each frame, and these sub-images are Fourier-transformed. (f) The FT magnitude is calculated, interpolated onto a logarithm-basis, and separated into four sub-images. (g) Each sub-image is Fourier-transformed and convolved with a phase filter. (h) Ensemble phase correlation is performed, producing a correlation plane with a peak shifted from the plane center. This shift corresponds to a frame pair strain rate. (i) Strain is computed by temporally integrating the strain rate estimates.
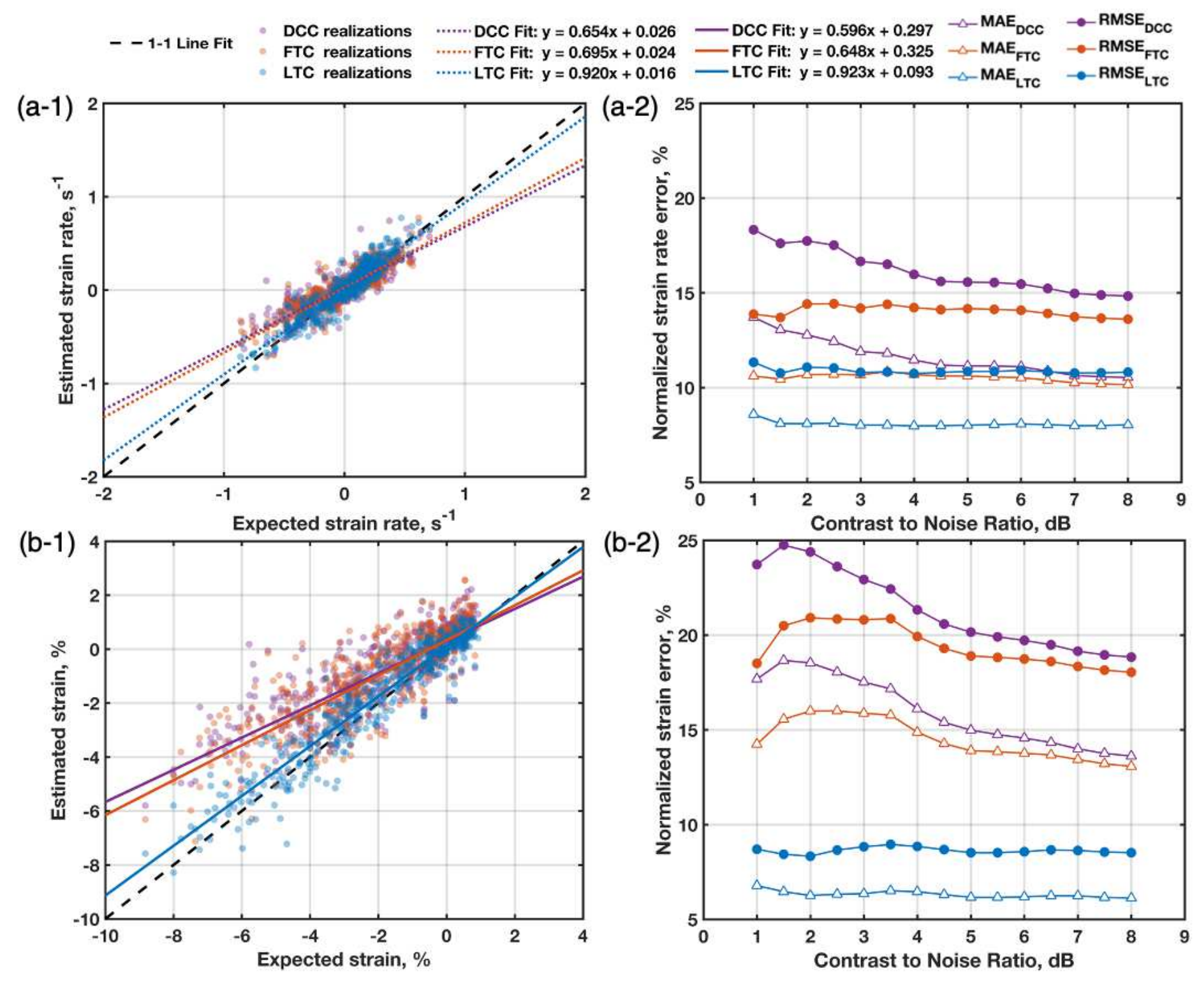

Figure 2

(Left) Direct comparison of measurements to ground truth values and (right) normalized mean absolute error (MAE) and root mean square error (RMSE) as a function of contrast-to-noise ratio (CNR) for (a) GLSr and (b) GLS quantities. Measurements were performed using the Direct Cross-Correlation method (DCC), Fourier Transform Correlation (FTC), and Fourier-based Logarithm Transform Correlation (LTC). 

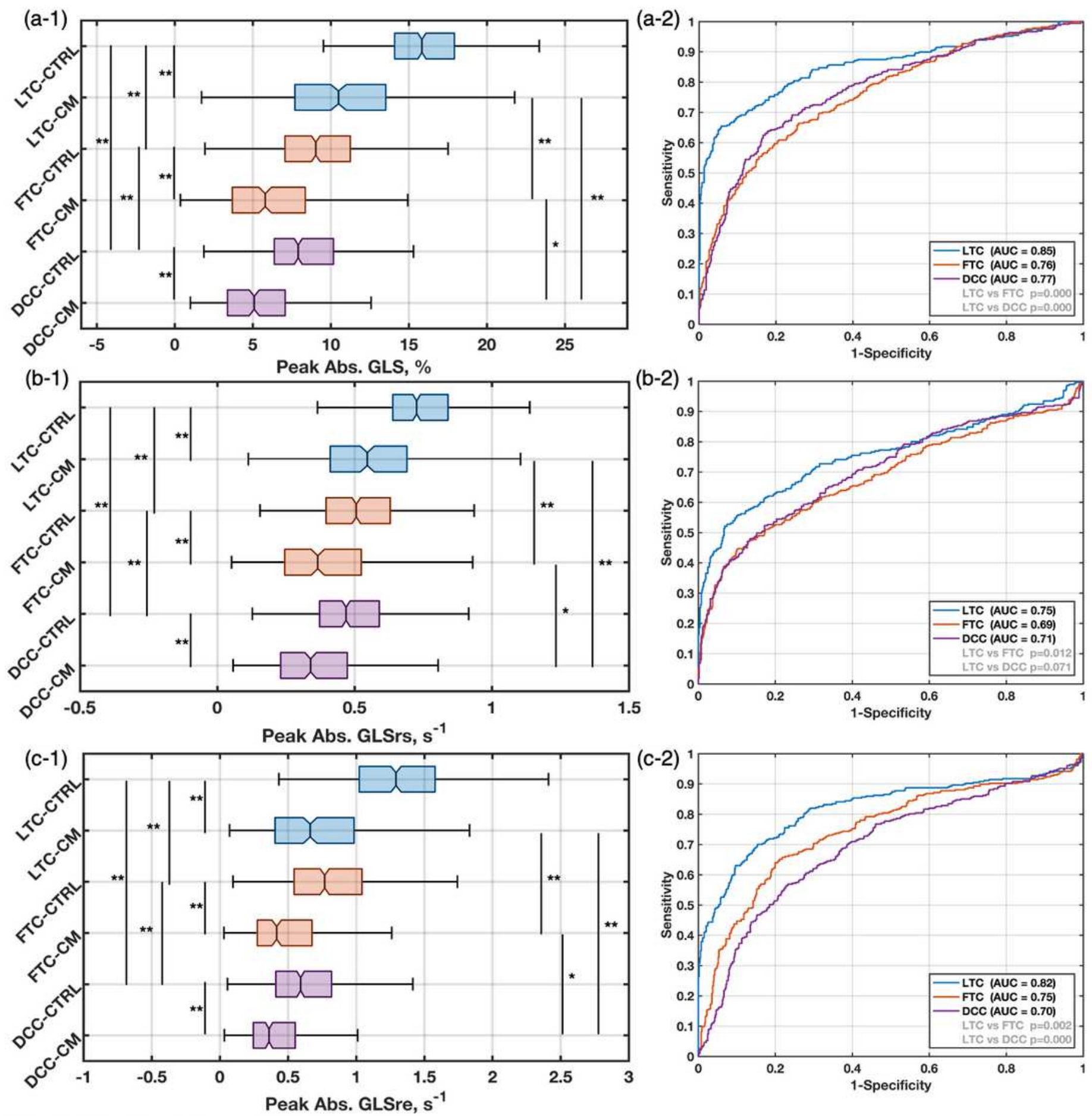

NS - Not Significant, ${ }^{\star}-p<0.050,{ }^{\star \star}-p<0.001$

\section{Figure 3}

Distribution of measurements and significance tests for each GLS measurement method on observing (a1) peak absolute GLS, (b-1) peak absolute systolic GLSr, and (c-1) peak absolute diastolic GLSr. (2) Receiver operating characteristic (ROC) curves displaying the ability for the LTC method estimated parameters to distinguish between normal and abnormal cardiac disease states based on (a-2) peak 
absolute GLS, (b-2) peak absolute systolic GLSr, and (c-2) peak absolute diastolic GLSr. The analysis was performed on a set of control subjects (CTRL) and subjects with cardiomyopathies (CM). 